\title{
Effect of Gain-Dependent Phase Shift on Fiber Laser Synchronization
}

\author{
Kurt Wiesenfeld, Slaven Peles, and Jeffrey L. Rogers
}

(Invited Paper)

\begin{abstract}
Recent experiments have demonstrated synchronization of fiber laser arrays at low and moderate pump levels. It has been suggested that a key dynamical process leading to synchronized behavior is the differential phase shift induced by the gain media. We explore theoretically the role of this effect in generating inphase dynamics. We find that its presence can substantially enhance the degree of inphase stability to an extent that could be practically important. At the same time, our analysis shows that a gain-dependent phase shift is not a necessary ingredient in the dynamical selection of the inphase state, thus, leading us to reconsider the essential mechanism behind inphase selection in fiber laser arrays.
\end{abstract}

Index Terms-Coupled oscillator systems, dynamics, laser arrays, phase-locked oscillators, phase synchronization, synchronization.

\section{INTRODUCTION}

$\mathbf{S}$ EVERAL recent experiments have reported encouraging results on an old problem: generating coherent radiation from an array of lasers. These experiments [1]-[9] use fiber lasers in various configurations. In this paper, we are concerned with arrays operating in the high-gain, high-loss limit that reported combined coherent radiation as high as $200 \mathrm{~W}$ [8], [9].

Despite some success in the laboratory and some success in modeling these systems [10]-[13], the key mechanism responsible for the synchronized behavior is still in question. Besides the obvious fundamental scientific interest, understanding the mechanism may be important if one ever hopes to achieve synchronization for very large arrays [14]-[16]. Indeed, experiments to date have reported array performance that systematically degrades for large arrays and at higher pump powers. A possible explanation for this degradation is that the key dynamical interaction becomes saturated at high pump levels. It has been suggested [17] that this key interaction is the phase shift resulting from the gain-dependent refractive index within each fiber.

Here, we present the first theoretical analysis of this effect in achieving synchronization in fiber laser arrays. We take as our

Manuscript received October 20, 2008; revised November 21, 2008. First published February 3, 2009; current version published April 8, 2009. This work was supported in part by the High Energy Laser Joint Technology Office and by the U.S. Army Research Office under Award W911NF-05-1-0506.

K. Wiesenfeld is with the School of Physics, Georgia Institute of Technology, Atlanta, GA 30332 USA (e-mail: kurt.wiesenfeld@ physics.gatech.edu).

S. Peles is with the United Technologies Research Center, East Hartford, CT 06108 USA (e-mail: peless@utrc.utc.com).

J. L. Rogers is with the Control and Dynamical Systems, California Institute of Technology, Pasadena, CA 91125 USA (e-mail: jeff@cds.caltech.edu).

Digital Object Identifier 10.1109/JSTQE.2008.2011491

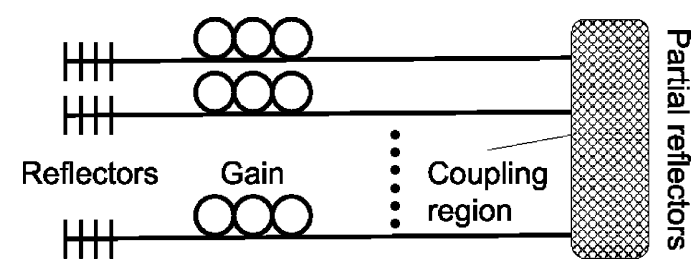

Fig. 1. Schematic of linear fiber laser arrays being studied. Output occurs at the partial reflectors.

starting point the coupled map model [13]

$$
\begin{aligned}
E_{n}(t+T)= & r \sum_{\ell=1}^{N} S_{n \ell} e^{G_{\ell}(t)} \sum_{m=1}^{N} S_{\ell m} E_{m}(t) \\
G_{n}(t+T)= & G_{n}(t)+\epsilon\left[W^{p} \tau\left(G_{\mathrm{tot}}-G_{n}(t)\right)-G_{n}(t)\right] \\
& -\frac{\epsilon}{I_{\text {sat }}}\left(1-e^{-2 G_{n}(t)}\right)\left|E_{n}(t)\right|^{2}
\end{aligned}
$$

that describes the evolution over one round-trip of the complex electric field amplitudes $E_{n}$ and real gains $G_{n}$ in the $n$th fiber. The reference plane is the coupler output face, $r$ is the output reflectivity coefficient, $S$ is the coupling matrix, $\epsilon$ is the ratio of round-trip time $T$ to the fluorescence time $\tau, W^{p}$ is the pump rate, $G_{\text {tot }}$ is the total available gain, and $I_{\text {sat }}$ is the field saturation parameter. This model is a refined version of the one introduced in [10] appropriate for four-level lasers; we will consider the three-level case later in the paper. This model has been shown to accurately reproduce various experimental observations reported for single- and coupled-fiber systems [13], [18]. A schematic of the system is shown in Fig. 1.

The model equations assume that the individual lasers are identical; our analysis will also assume a lossless coupler. While the effects of disorder are important in developing a full understanding of the system, this idealized limit captures much of the essential physics and has the added advantage of being analytically tractable. Moreover, our present goal is to explore, in particular, the effect of a gain-dependent refractive index, and from this perspective, a relative comparison of the system dynamics is appropriate.

Our first step is to modify this model to include the effect of gain-dependent phase shift. In the derivation given in [10], the amplification of the electromagnetic wave as it passes through the gain medium is represented by the exponential factor in (1). The physical basis for the new effect is that the index of refraction of the fiber depends on the gain. While passing through the gain region, the electromagnetic wave suffers a change in propagation speed due to the change in the refractive index. The 
change in propagation speed leads to a phase shift above and beyond that due to free-space propagation. For a single fiber, the additional phase shift can be absorbed in the overall phase factor and is consequently irrelevant for the dynamics. But for two or more lasers, these phase shifts become relevant since they typically lead to a relative phase change between fiber fields, and this is crucial when considering synchronization properties.

Mathematically, this physical effect may be modeled by making the replacement

$$
\exp \left(G_{\ell}\right) \rightarrow \exp \left(\xi G_{\ell}\right)
$$

where $\xi$ is a new, complex parameter. In what follows, we consider the case of lossless, global coupling. The plan is to investigate the stability of the inphase solution, and especially, the effect of the imaginary part of the new parameter. To this end, we set

$$
\xi=1+\imath \theta
$$

with $\theta$ being real.

\section{Continuous-Wave (CW) InPhase State AND Stability}

To simplify notation, we write $E$ for $E(t)$ and $E^{\prime}$ for $E(t+$ $T)$; it is also convenient to introduce the scaled field variables $E_{n} \rightarrow E_{n} \sqrt{I_{\mathrm{sat}}}$. The model becomes

$$
\begin{aligned}
E_{n}^{\prime}= & r \sum_{\ell=1}^{N} S_{n \ell} e^{\xi G_{\ell}} \sum_{m=1}^{N} S_{\ell m} E_{m} \\
G_{n}^{\prime}= & G_{n}+\epsilon\left[W^{p} \tau\left(G_{\mathrm{tot}}-G_{n}\right)-G_{n}\right] \\
& -\epsilon\left(1-e^{-2 G_{n}}\right)\left|E_{n}\right|^{2} .
\end{aligned}
$$

For global coupling, the matrix $S$ has the form

$$
S_{n m}=(a-b) \delta_{n m}+b
$$

with $a$ and $b$ being complex constants. This matrix has one nondegenerate eigenvalue $M$ and an $(N-1)$ fold degenerate eigenvalue $m$, given by

$$
\begin{aligned}
M & =a+(N-1) b \\
m & =a-b .
\end{aligned}
$$

In what follows, we consider the coupling produced by evanescent overlap of the individual laser fields, e.g., as occurs within a fused fiber coupler. We further assume that the coupler is lossless, in which case $\mathrm{S}$ is unitary [12]. This property of the coupling matrix $S$ requires both $M$ and $m$ to have unit modulus. Under these circumstances, there exist fully symmetric, constant intensity solutions [10]-[12]. Equations (4) and (5) can be rewritten to identify solutions using $E_{n}=\bar{E}$ and $G_{n}=\bar{G}$

$$
\begin{aligned}
\bar{E}^{\prime}= & r e^{\xi \bar{G}}\left(\sum_{\ell} \sum_{m} S_{n \ell} S_{\ell m}\right) \bar{E} \\
\bar{G}^{\prime}= & \bar{G}+\epsilon\left[W^{p} \tau\left(G_{\mathrm{tot}}-\bar{G}\right)-\bar{G}\right] \\
& -\epsilon\left(1-e^{-2 \bar{G}}\right)|\bar{E}|^{2} .
\end{aligned}
$$

Performing the double sum in (6)

$$
\bar{E}^{\prime}=r e^{\bar{G}}\left(e^{\imath \bar{G} \theta} M^{2}\right) \bar{E} .
$$

Transforming into a uniformly rotating frame

$$
\bar{E}=\left(e^{i \bar{G} \theta} M^{2}\right)^{t} \bar{F}
$$

we have

$$
\begin{aligned}
\bar{F}^{\prime}= & r e^{\bar{G}} \bar{F} \\
\bar{G}^{\prime}= & \bar{G}+\epsilon\left[W^{p} \tau\left(G_{\mathrm{tot}}-\bar{G}\right)-\bar{G}\right] \\
& -\epsilon\left(1-e^{-2 \bar{G}}\right)|\bar{F}|^{2} .
\end{aligned}
$$

This map admits the fixed point solution

$$
\begin{aligned}
\bar{G} & =\ln \left(\frac{1}{r}\right) \\
|\bar{F}|^{2} & =\frac{W^{p} \tau G_{\mathrm{tot}}-\left(W^{p} \tau+1\right) \ln (1 / r)}{1-r^{2}} .
\end{aligned}
$$

Note that the phase of $\bar{F}$ is arbitrary, as expected, because of the global phase-shift symmetry of the original equations (4) and (5). In terms of the $E$-field, this "on" fixed point solution is

$$
\bar{E}=M^{2 t} \bar{F} .
$$

Turning to the stability of this solution, set

$$
\begin{aligned}
& E_{n}=\bar{E}+\eta_{n} \\
& G_{n}=\bar{G}+\gamma_{n}
\end{aligned}
$$

where $\eta_{n}$ is complex and $\gamma_{n}$ is real. Upon substituting these into the original map, and ignoring higher order terms in the perturbations, we are led to the following linearized map:

$$
\begin{aligned}
\eta_{n}^{\prime}= & r e^{\xi \bar{G}} \xi \bar{E} M\left(m \gamma_{n}+b \sum_{\ell} \gamma_{\ell}\right) \\
& +r e^{\xi \bar{G}}\left(m^{2} \eta_{n}+d \sum_{\ell} \eta_{\ell}\right) \\
\gamma_{n}^{\prime}= & \left\{1-\epsilon\left(W^{p} \tau+1\right)-2 \epsilon e^{-2 \bar{G}}|\bar{E}|^{2}\right\} \gamma_{n} \\
& -\epsilon\left(1-e^{-2 \bar{G}}\right)\left(\bar{E} \eta_{n}^{\star}+\bar{E}^{\star} \eta_{n}\right)
\end{aligned}
$$

where $d$ is the off-diagonal element of the matrix $S^{2}$

$$
d=2 a b+(N-2) b^{2} .
$$

We introduce the sum and difference coordinates

$$
\begin{aligned}
H & =\sum_{n=1}^{N} \eta_{n} \\
h_{n} & =\eta_{n}-\eta_{n+1}, \quad n=1, \ldots, N-1 \\
\Gamma & =\sum_{n=1}^{N} \gamma_{n} \\
g_{n} & =\gamma_{n}-\gamma_{n+1}, \quad n=1, \ldots, N-1 .
\end{aligned}
$$

By summing (11) and (12) over all $n$ and noting that

$$
\sum_{n}\left[m \gamma_{n}+b \sum_{\ell} \gamma_{\ell}\right]=m \Gamma+N b \Gamma=M \Gamma
$$


and

$$
\sum_{n}\left[m^{2} \eta_{n}+d \sum_{m} \eta_{m}\right]=m^{2} H+N d H=M^{2} H
$$

we arrive at a decoupled subsystem of equations for $(H, \Gamma)$, namely

$$
\begin{aligned}
H^{\prime}= & r e^{\xi \bar{G}} M^{2}(\xi \bar{E} \Gamma+H) \\
\Gamma^{\prime}= & \left\{1-\epsilon\left(W^{p} \tau+1\right)-2 \epsilon e^{-2 \bar{G}}|\bar{E}|^{2}\right\} \Gamma \\
& -\epsilon\left(1-e^{-2 \bar{G}}\right)\left(\bar{E} H^{\star}+\bar{E}^{\star} H\right) .
\end{aligned}
$$

Meanwhile, by taking differences between the $n$th and $(n+1)$ th stability equations (11) and (12), we get the $(N-1)$ decoupled subsystems

$$
\begin{aligned}
h_{n}^{\prime}= & r e^{\xi \bar{G}} \bar{E} M m g_{n}+r e^{\xi \bar{G}} m^{2} h_{n} \\
g_{n}^{\prime}= & \left\{1-\epsilon\left(W^{p} \tau+1\right)-2 \epsilon e^{-2 \bar{G}}|\bar{E}|^{2}\right\} g_{n} \\
& -\epsilon\left(1-e^{-2 \bar{G}}\right)\left(\bar{E} h_{n}^{\star}+\bar{E}^{\star} h_{n}\right) .
\end{aligned}
$$

For the constant intensity solution, we have

$$
r e^{\bar{G}}=1 \quad \text { and } \quad|\bar{E}|^{2}=|\bar{F}|^{2}
$$

so that these become [see (3)]

$$
\begin{aligned}
H^{\prime}= & e^{\imath \theta \bar{G}} M^{2}(\xi \bar{E} \Gamma+H) \\
\Gamma^{\prime}= & \left\{1-\epsilon\left(W^{p} \tau+1+2 r^{2}|\bar{F}|^{2}\right)\right\} \Gamma \\
& -\epsilon\left(1-r^{2}\right)\left(\bar{E} H^{\star}+\bar{E}^{\star} H\right)
\end{aligned}
$$

and

$$
\begin{aligned}
h_{n}^{\prime}= & e^{\imath \theta \bar{G}} \bar{E} M m g_{n}+e^{\imath \theta \bar{G}} m^{2} h_{n} \\
g_{n}^{\prime}= & \left\{1-\epsilon\left(W^{p} \tau+1+2 r^{2}|\bar{F}|^{2}\right)\right\} g_{n} \\
& -\epsilon\left(1-r^{2}\right)\left(\bar{E} h_{n}^{\star}+\bar{E}^{\star} h_{n}\right) .
\end{aligned}
$$

The following transformation simplifies matters by eliminating the time dependence of the coefficients (appearing via $\bar{E}$ )

$$
H=\left(e^{\imath \theta \bar{G}} M^{2}\right)^{t} \tilde{H} \quad h_{n}=\left(e^{\imath \theta \bar{G}} M^{2}\right)^{t} \tilde{h}_{n}
$$

so that, recalling (10), we get

$$
\begin{aligned}
\tilde{H}^{\prime}= & \xi \bar{F} \Gamma+\tilde{H} \\
\Gamma^{\prime}= & \left\{1-\epsilon\left(W^{p} \tau+1+2 r^{2}|\bar{F}|^{2}\right)\right\} \Gamma \\
& -\epsilon\left(1-r^{2}\right)\left(\bar{F} \tilde{H}^{\star}+\bar{F}^{\star} \tilde{H}\right)
\end{aligned}
$$

and similarly

$$
\begin{aligned}
\tilde{h}_{n}^{\prime}= & \xi \bar{F} M^{\star} m g_{n}+\left(M^{\star} m\right)^{2} \tilde{h}_{n} \\
g_{n}^{\prime}= & \left\{1-\epsilon\left(W^{p} \tau+1+2 r^{2}|\bar{F}|^{2}\right)\right\} g_{n} \\
& -\epsilon\left(1-r^{2}\right)\left(\bar{F} \tilde{h}_{n}^{\star}+\bar{F}^{\star} \tilde{h}_{n}\right) .
\end{aligned}
$$

To test the stability, we need the eigenvalues of each of these subsystems. The base state is stable if all of the eigenvaluesexcluding the single unity eigenvalue forced by the overall phase-shift symmetry-lie inside the unit circle. Consider first the $(\tilde{H}-\Gamma)$ subsystem. We want the eigenvalues of

$$
\left(\begin{array}{ccc}
1 & 0 & \xi \bar{F} \\
0 & 1 & \xi^{\star} \bar{F}^{\star} \\
v^{\star} & v & 1-w
\end{array}\right)
$$

where

$$
\begin{aligned}
v & =-\epsilon\left(1-r^{2}\right)|\bar{F}| \\
w & =\epsilon\left(W^{p} \tau+1+2 r^{2}|\bar{F}|^{2}\right) .
\end{aligned}
$$

One of the three eigenvalues is exactly 1 (the symmetry-forced eigenvalue); the other two are the roots $\mu$ of the quadratic

$$
(1-\mu)(1-w-\mu)-\left(v^{\star} \bar{F}+v \bar{F}^{\star}\right)=0 .
$$

This depends on the base solution only through the singlefiber intensity $I=|\bar{F}|^{2}$, which depends linearly on the pump $W^{p} \tau$, increasing from zero at onset. Just above onset, the roots $\mu$ lie inside the unit circle (initially, they are real; for larger $W^{p} \tau$, they are complex conjugates) until the critical value

$$
\bar{I}_{\text {hopf }}=\frac{W^{p} \tau+1}{2-4 r^{2}} .
$$

The other set of stability eigenvalues is determined by the $\left(\tilde{h}_{n}-g_{n}\right)$ subsystems, i.e., these are the eigenvalues of the matrix

$$
\left(\begin{array}{ccc}
\left(M^{\star} m\right)^{2} & 0 & \xi \bar{F} M^{\star} m \\
0 & \left(M m^{\star}\right)^{2} & \xi^{\star} \bar{F}^{\star} M m^{\star} \\
v^{\star} & v & 1-w
\end{array}\right)
$$

where $v$ and $w$ are as before.

The eigenvalues are roots of a cubic polynomial. The effect of the coupler appears through the two parameters $M$ and $m$; in fact, we have the interesting and useful result that the eigenvalues depend on the coupling only through the single (real) quantity $M^{\star} m+M m^{\star}$. Since $M$ and $m$ have unit modulus, so does $M^{\star} m$, and the coupling parameter can be taken as the phase of this product, which we will call $\zeta$. We have

$$
M^{\star} m+M m^{\star}=e^{i \zeta}+e^{-i \zeta}=2 \cos \zeta .
$$

Note that when the coupling matrix $\mathrm{S}$ is the identity so that the coupler is effectively absent, the coupling parameter $\zeta$ is zero. In this sense, one can view $|\zeta|$ as a measure of the coupling strength, at least for small values.

\section{EfFect of GaIn-DePendent Phase ShIfTs}

We now consider the stability properties of the inphase state with and without the presence of the gain-dependent phase shift. Consider first the unmodified model so that the parameter $\theta$ is zero. Fig. 2 shows the modulus of the largest magnitude eigenvalue, plotted as a function of coupler parameter $\zeta$ and pump strength $W^{p} \tau$. The former runs over its full range from 0 to $2 \pi$ and the latter runs over its full range from the onset of lasing to the previously determined Hopf point (where the solution loses stability within the symmetric subspace). The plots are 

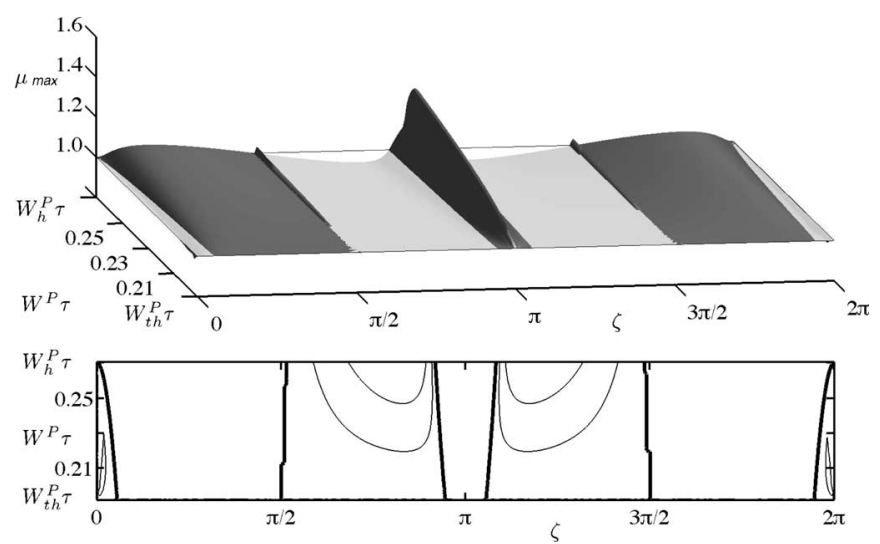

Fig. 2. Surface (top) and contour (bottom) plots of the largest magnitude eigenvalue for the "difference" subspace matrix (13) as a function of the coupling parameter $\zeta$ and the pump parameter $W^{p} \tau$; the other parameters are: $G_{\text {tot }}=$ $10, r=0.2, \epsilon=0.1$, and $\theta=0$. Values below unity are stable; the plane bisects the surface at 1 resulting in the inphase state being stable in the light gray regions (below the plane) and unstable in the dark gray regions (above the plane).
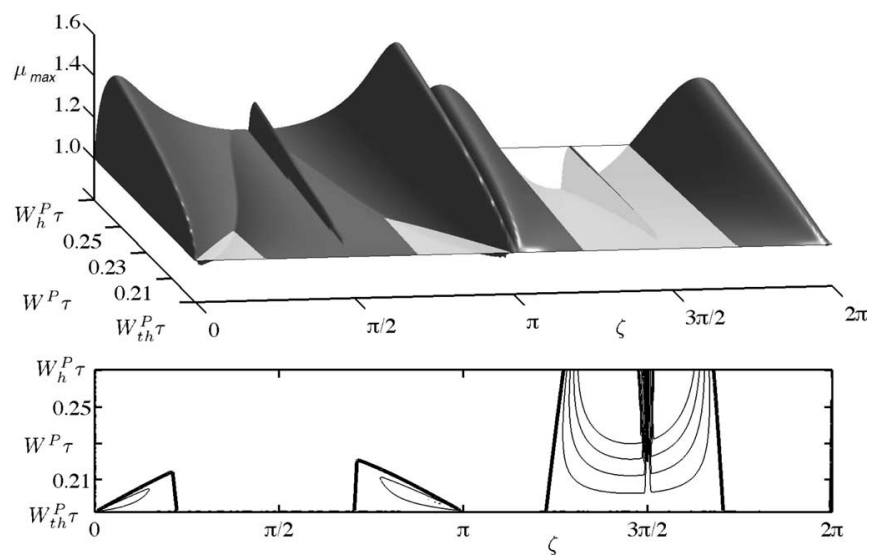

Fig. 3. Same as previous figure, but with $\theta=4$.

symmetric about $\zeta=\pi$; there is a narrow window of stability centered around $\zeta=0$ and broad windows of stability roughly centered on $\zeta=3 \pi / 4$ and $\zeta=5 \pi / 4$. The narrow glitches that appear close to $\zeta=\pi / 2$ and $\zeta=3 \pi / 2$ are genuine (not artifacts); however, since they lie outside the stability windows, they are not relevant to our present purpose.

Now consider how the complex nature of $\xi$ modifies these results. Fig. 3 shows the leading eigenvalue for $\theta=4$ with all other parameters as before. We pick this value because it seems to give the greatest difference with the previous plot. The stability boundaries (corresponding to the contour value of one) have shifted substantially relative to the earlier case $(\theta=0)$. Although the larger stability window has narrowed somewhat, the most stable operating point is significantly more stable (the minimum value of $\mu_{\max }$ is about five times as far below unity as the $\theta=0$ case).

\section{STABILITY BOUNDARIES}

In this section, we derive analytic expressions for the bifurcation curves. In the lower panes of Figs. 2 and 3 these are the dark contours corresponding to the value $\mu_{\max }=1$. We concern ourselves with the range of pump parameter $W^{p} \tau$ above onset of the CW state and below the inphase Hopf point at which pulsing sets in. The problem then amounts to considering the eigenvalues $\mu$ of the matrix (13). It is convenient to write this matrix as

$$
\left(\begin{array}{ccc}
a & 0 & b \\
0 & a^{\star} & b^{\star} \\
\epsilon c & \epsilon c & 1-\epsilon d
\end{array}\right)
$$

where

$$
\begin{aligned}
a & =\left(M^{\star} m\right)^{2} \\
b & =\xi \bar{F} M^{\star} m \\
c & =-\left(1-r^{2}\right) \bar{F} \\
d & =W^{p} \tau+1+2 r^{2} \bar{F}^{2} .
\end{aligned}
$$

In writing the matrix this way, we have taken $\bar{F}$ to be real, which is valid since the entire family of solutions (which all share the same value of $|\bar{F}|$ ) has the same set of eigenvalues. The characteristic equation is

$$
\begin{aligned}
& (a-\mu)\left(a^{\star}-\mu\right)(1-\epsilon d-\mu) \\
& \quad-\epsilon b c\left(a^{\star}-\mu\right)-\epsilon b^{\star} c(a-\mu)=0 .
\end{aligned}
$$

Consider first the case of a Hopf bifurcation. Equation (16) is equivalent to

$$
\left(\mu_{1}-\mu\right)\left(\mu_{2}-\mu\right)\left(\mu_{3}-\mu\right)=0
$$

where $\mu_{j}$ represent the three eigenvalues. At a Hopf point, we have $\mu_{1}=\mu_{2}^{\star}$ and $\left|\mu_{1}\right|^{2}=1$. If we expand the previous equation and use these two conditions, we get

$$
-\mu^{3}+\left(\nu+\mu_{3}\right) \mu^{2}-\left(1+\nu \mu_{3}\right) \mu+\mu_{3}=0
$$

where $\nu=2 \operatorname{Re} \mu_{1}$. If we expand (16) and equate the corresponding polynomial coefficients, we get the following three equations (recall that $a a^{\star}=1$ ):

$$
\begin{aligned}
\nu+\mu_{3} & =a+a^{\star}+1-\epsilon d \\
\nu \mu_{3} & =\epsilon\left(b+b^{\star}\right) c-\left(a+a^{\star}\right)(1-\epsilon d) \\
\mu_{3} & =1-\epsilon d-\epsilon c\left(b a^{\star}+b^{\star} a\right) .
\end{aligned}
$$

We can eliminate $\mu_{3}$ and $\nu$ to arrive at a single equation

$$
1-\epsilon d-\epsilon c\left(b a^{\star}+b^{\star} a\right)-\left(a+a^{\star}\right)=-\frac{b+b^{\star}}{b a^{\star}+b^{\star} a} .
$$

To within order $\epsilon$, then we get the bifurcation condition by balancing the order unity terms

$$
1-\left(a+a^{\star}\right)=-\frac{b+b^{\star}}{b a^{\star}+b^{\star} a}
$$

so that, upon setting [cf., (14)]

$$
\begin{aligned}
a & =\left(M^{\star} m\right)^{2}=e^{2 i \zeta} \\
b & =\xi \bar{F} M^{\star} m=\rho e^{i \beta} \\
1-2 \cos 2 \zeta & =-\frac{\cos \beta}{\cos (\beta-2 \zeta)} .
\end{aligned}
$$


Note that $\beta$ is unaffected by the value of $\bar{F}$ so that for a fixed value of the parameter $\xi$, (20) is independent of the pump strength $W^{p} \tau$. Ploting in the $\left(W^{p} \tau, \zeta\right)$ plane, the stability boundaries corresponding to the Hopf bifurcation show up as lines of constant $W^{p} \tau$ (vertical lines in plots like the bottom panels of Figs. 2 and 3). Corrections of order $\epsilon$ modify the lines slightly.

Now consider the bifurcation(s) corresponding to a real eigenvalue crossing +1 . Direct evaluation of the characteristic equation (16) with $\mu=1$ yields, after a little algebra,

$$
2 d-\left(a+a^{\star}\right) d-c\left[\left(b+b^{\star}\right)-\left(b a^{\star}+b^{\star} a\right)\right]=0
$$

where we have used $a^{\star} a=1$. Substituting the explicit expressions for $a, b, c$, and $d$ yields the desired condition

$$
\bar{F}^{2}=\frac{W^{p} \tau+1}{\left(1-r^{2}\right)(\theta / \sin \zeta)-2 r^{2}} .
$$

Note that if $\theta=0$, this condition is never satisfied. In this sense (and this sense alone), inclusion of the gain-dependent refractive index leads to a fundamentally new feature.

Recall that $\bar{F}^{2}$ is just the intensity of the electric field and that (for the constant gain solutions we are analyzing) it is a linear function of the pump parameter $W^{p} \tau$. At onset, $\bar{F}$ is zero and increases with the pump up until the onset of gain oscillations that occur at

$$
\bar{F}^{2}=\frac{W^{p} \tau+1}{2-4 r^{2}} .
$$

For Figs. 2 and 3,r $=0.2$, and so this (largest) value of $\bar{F}^{2}$ is a bit under 0.6. The final bifurcation type to consider is period doubling, where a real eigenvalue exits the unit circle at -1 . Setting $\mu=-1$ in (16) and using $a^{\star} a=1$ yields

$$
0=\left(2+a+a^{\star}\right)(2-\epsilon d)-\epsilon\left(b+b^{\star}\right) c-\epsilon c\left(b a^{\star}+b^{\star} a\right) .
$$

This has a solution only if

$$
2+a+a^{\star}=O(\epsilon) .
$$

With $a=\exp (2 i \zeta)$, it follows that period-doubling bifurcations occur at

$$
\zeta=\frac{\pi}{2}, \frac{3 \pi}{2}
$$

for $\zeta \in[0,2 \pi]$. In fact, one readily shows that the $O(\epsilon)$ corrections vanish identically so that the aforementioned bifurcation lines are good up to $O\left(\epsilon^{2}\right)$.

The upper panel of Fig. 4 plots the derived bifurcation curves in the $W^{p} \tau-\zeta$ plane, using the same parameter values as those in Fig. 3. Condition (20) yields six vertical lines, while condition (21) yields two inclined segments. The period doubling at $\zeta=3 \pi / 2$ shows up as a thin sliver in the stability diagram (see Fig. 4). The other $(\zeta=\pi / 2)$ is "invisible" because there is another eigenvalue with even larger magnitude (and thus, the base solution is already unstable). These results should be compared against the lower panel that reproduces the critical contour of Fig. 3, i.e., the contour for $\left|\mu_{\max }\right|=1$. The agreement is excellent. Our analysis is good to order $\epsilon$, and we have used the

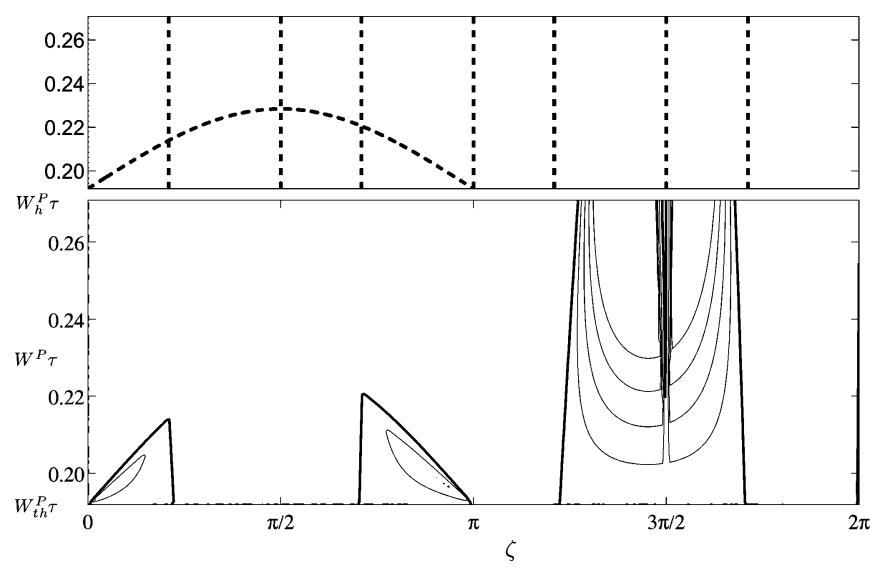

Fig. 4. Stability boundaries of the inphase fixed point as a function of $W^{p} \tau$ and $\zeta$. The upper panel uses the perturbative analytic formulas derived in the text; the lower panel reproduces the critical contour from Fig. 3.

value $\epsilon=0.1$ in the figure. The agreement improves with more physically typical values $\epsilon \sim 10^{-4}$.

\section{RESUlTS FOR THREE-LEVEL LASERS}

The results derived earlier are based on the equations describing four-level lasers. For completeness, we quote the results for the three-level case. In the governing dynamical equations, one must replace (5) with [13]

$$
\begin{aligned}
G_{n}^{\prime}= & G_{n}+\epsilon\left[W^{p} \tau\left(G_{\mathrm{tot}}-G_{n}\right)-\left(G_{\mathrm{tot}}+G_{n}\right)\right] \\
& -2 \epsilon\left(1-e^{-2 G_{n}}\right)\left|E_{n}\right|^{2} .
\end{aligned}
$$

The analysis proceeds exactly as before and yields the following results. The inphase $\mathrm{CW}$ state is

$$
\begin{aligned}
\bar{G} & =\ln \left(\frac{1}{r}\right) \\
|\bar{F}|^{2} & =\frac{W^{p} \tau G_{\mathrm{tot}}-\left(W^{p} \tau+1\right) \ln (1 / r)}{2\left(1-r^{2}\right)} .
\end{aligned}
$$

This state loses stability within the inphase subspace when the intensity reaches a value

$$
\bar{I}_{\text {hopf }}=\frac{W^{p} \tau+1}{4-8 r^{2}} .
$$

In the regime $0<\bar{I}<\bar{I}_{\text {hopf }}$, the $\mathrm{CW}$ inphase state may be either stable or unstable, depending on the eigenvalues of the symmetry-broken subspace. These are determined by the characteristic equation (16), where now

$$
\begin{aligned}
a & =\left(M^{\star} m\right)^{2} \\
b & =\xi \bar{F} M^{\star} m \\
c & =-2\left(1-r^{2}\right) \bar{F} \\
d & =W^{p} \tau+1+4 r^{2} \bar{F}^{2} .
\end{aligned}
$$


The conditions for Hopf, saddle-node, and period-doubling instabilities are, respectively

$$
\begin{aligned}
1-2 \cos 2 \zeta & =-\frac{\cos \beta}{\cos (\beta-2 \zeta)} \\
\bar{F}^{2} & =\frac{W^{p} \tau+1}{2\left(1-r^{2}\right)(\theta / \sin \zeta)-4 r^{2}} \\
\zeta & =\frac{\pi}{2}, \frac{3 \pi}{2}
\end{aligned}
$$

where $\zeta$ is given by (14) and $\beta$ by (19).

\section{Physical Mechanism Behind Coherence}

Our analysis shows that there exist wide regions of inphase stability, and this property persists with or without explicit introduction of a gain-dependent phase shift (embodied by a nonzero imaginary part $\theta$ of the parameter $\xi$ ). We did find that a gaindependent phase shift can strengthen the fixed point stability, and as such it might play a significant role if one were to include the effects of noise or other imperfections in array properties. But this begs the question: if a gain-dependent phase shift is not a necessary ingredient, then what is the essential physical mechanism behind the stability of the inphase state? As we now describe, the answer lies in the dynamical interplay between coupling and gain dynamics.

The essential physics is contained in the simplest case of two lasers, and for the sake of clarity, we restrict our discussion to this case. We can also take advantage of the system architecture: since the gain dynamics and the coupling dynamics take place in different parts of the system, we can treat the two effects sequentially rather than simultaneously over each round-trip.

Imagine first the situation without the coupler. Then, each laser operates independently, settling into a steady state with fixed intensity and gain; for identical fibers, these values are the same in each laser. The relative phase of the $E$-fields is completely free with no tendency for inphase, antiphase, or any other dynamical state.

The effect of turning on the coupler is to introduce beating: waves that enter the coupler with equal amplitudes but unequal phases emerge with unequal amplitudes. Since the coupler is lossless, this beating would continue indefinitely if not for the dissipative gain dynamics. As the unequal $E$-fields pass through the respective gain regions of the two fibers, the initially equal gains react differently: amplifying their respective $E$-fields by the same factor, one becomes overly depleted and the other underdepleted. As a result, the beating of the $E$-fields within the coupler induces beating of the gains as well.

The situation is illustrated in Fig. 5, which shows the resulting time series when an initially uncoupled, steady-state system is given a perturbation away from the inphase state that advances the phase of one $E$-field by a $0.4 \mathrm{rad}$ and retards the phase of the other by an equal amount.

As the energy sloshes back and forth between the fibers, the controlling factor is the degree of beating induced by the coupler. Since the coupler is lossless, its only characteristic property is the amount of mode mixing per pass. This is the quantity that appears in our analysis as $\zeta$. Over some range of $\zeta$, the beating
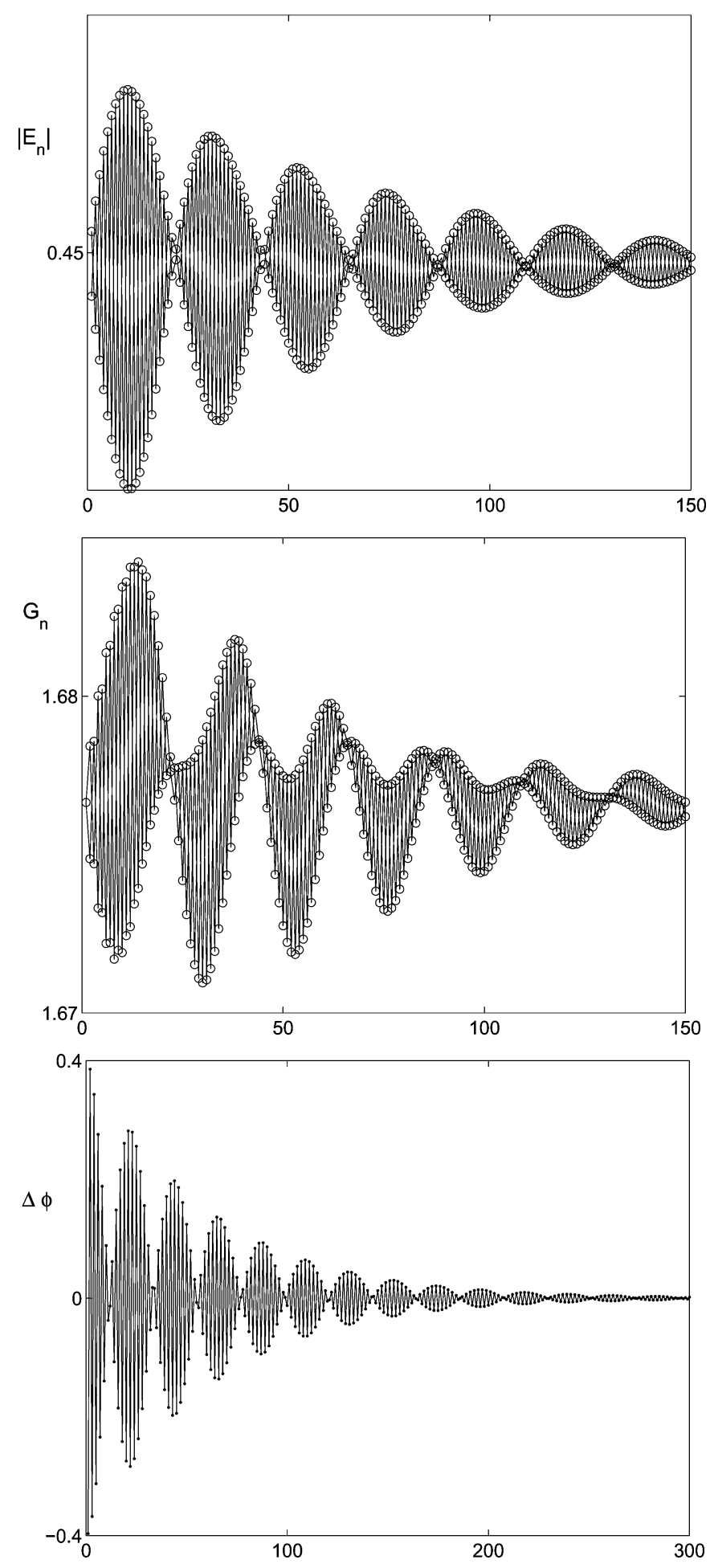

Fig. 5. Time series for (top to bottom) $\left|E_{n}\right|, G_{n}$, and relative phases of a two-laser system. Each laser is initially in its own steady state, the initial phase difference is $0.8 \mathrm{rad}$. Other parameters are $W^{p} \tau=0.2, G_{\text {tot }}=10, r=$ $0.2, \zeta=1.6, \epsilon=0.1$, and $\theta=0$

leads to a slow but inexorable damping out of the antiphase perturbation; otherwise, the antiphase perturbation slowly grows. (The only equal amplitude states for which beating is absent are the inphase and antiphase states.) Which of these tendencies "wins" depends on the relation between the two beating entities, i.e., the $E$-fields and the gains. We note that if the gain had 

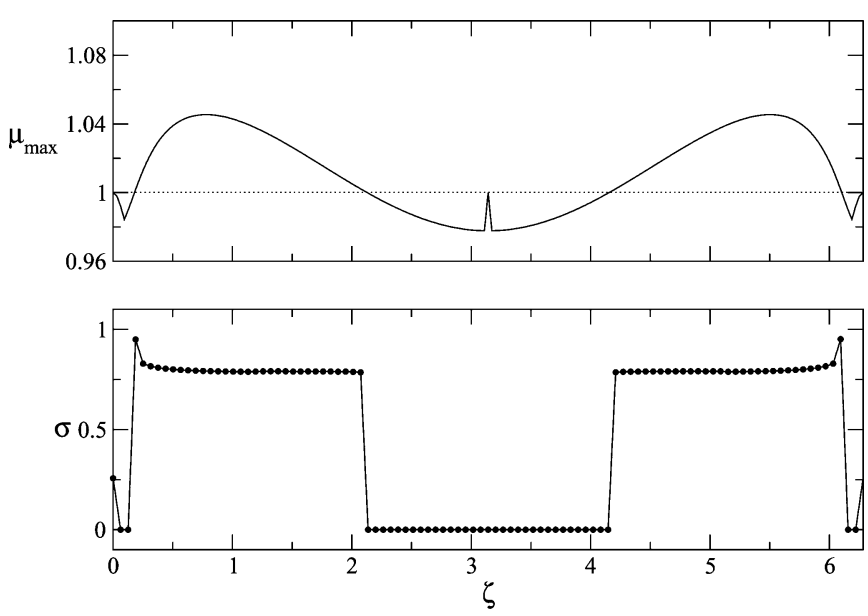

Fig. 6. (Top) Leading eigenvalue of matrix (13) for the inphase fixed point as a function of coupler parameter $\zeta$. (Bottom) Synchronization measure $\sigma$ determined from numerical simulations of three lasers. The parameter $\theta$ is zero.

no dynamical character-i.e., it entered the evolution equations strictly as a parameter-inphase stability would depend on $\zeta$ alone, independent of the other parameters, corresponding to strictly vertical stability boundaries in the $W^{p} \tau-\zeta$ plane.

\section{NUMERICAL RESULTS}

The analysis in this paper is restrictive in two respects. First, we have considered only the constant intensity, constant gain inphase state; second, we have determined only the local stability of that state. To extend the theory in either respect is, from an analytic point of view, notoriously difficult. In this section, we present a summary of some numerical results we generated by direct simulation of the governing dynamical equations. As with our earlier investigations of fiber laser arrays [10]-[12], we find that the derived properties of the $\mathrm{CW}$ state provide a useful guide even as the system displays far more complicated dynamics, though some differences arise. We have no deep understanding of this apparent correspondence. The following data are presented in the spirit of providing a more rounded picture of the system's behavior.

We ran a large set of simulations of (4) and (5) for an array of three fibers, using randomly chosen initial conditions and various parameter values. Typically, the observed dynamical behavior was complicated, and as a measure of the degree of synchronization, we used the quantity $\sigma$ defined by

$$
\sigma=\left\langle\frac{\left|\sum_{i} E_{i}\right|}{\sum_{i}\left|E_{i}\right|}\right\rangle_{t}
$$

where $\langle\cdots\rangle$ denotes a long-time average. This quantity achieves its maximum value of unity just in case the system is in a perfectly inphase state, it diminishes as the quality of synchronization degrades, and its smallest possible value is zero.

Fig. 6 summarizes the results of a set of simulations without the gain-dependent phase shift (so $\theta=0$ ). The pump strength was held fixed at $W^{p} \tau=0.24$ and the coupling parameter $\zeta$ was varied over its full range from 0 to $2 \pi$. The other parameters are the same as in Fig. 2. The top panel shows the leading
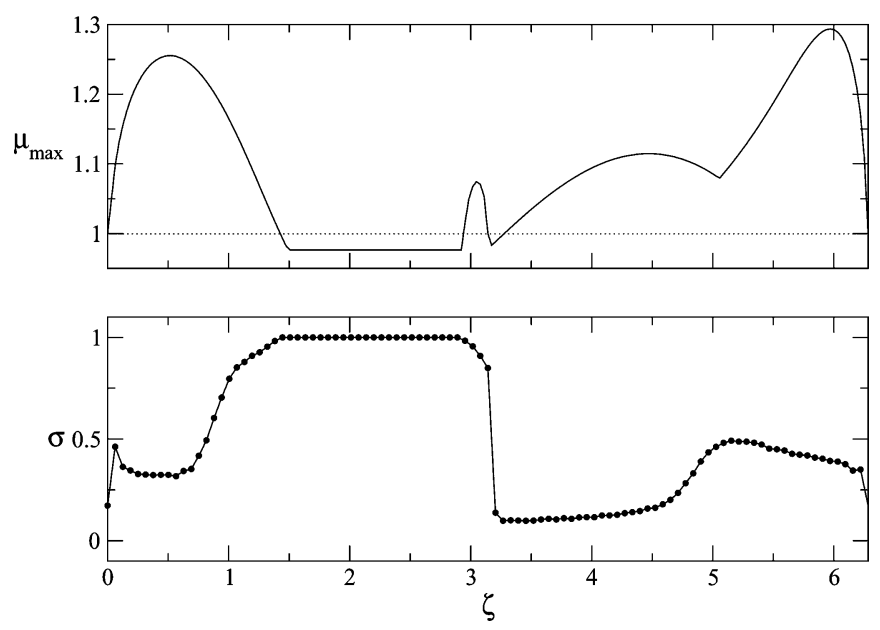

Fig. 7. Same as Fig. 6 but with $\theta=3 \pi / 2$.
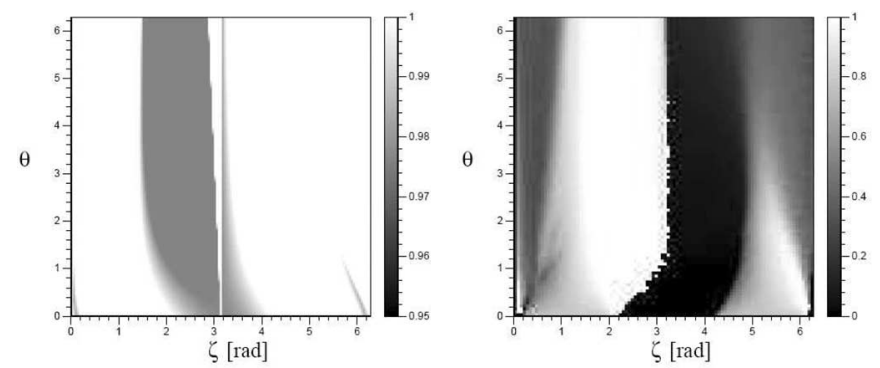

Fig. 8. Leading eigenvalue $\mu_{\max }$ of the inphase fixed point (left panel) and synchronization measure $\sigma$ (right panel) as a function of $\zeta$ and $\theta$.

eigenvalue of the inphase fixed point and the bottom panel shows the synchronization parameter $\sigma$. At first blush, one might expect to see $\sigma=1$ whenever $\mu_{\max }$ dips below unity since the latter implies that the inphase fixed point is stable. But it happens that the basin of attraction of the inphase state is very small [12], and for a typical initial condition, the system evolves into a poorly synchronized state [11]. In contrast, we see that when the inphase fixed point is locally unstable, the array behavior may be complicated but is reasonably synchronized. We see that in this case the results of the local stability analysis are not a good reflection of the global dynamical behavior.

The situation is dramatically different when the gaindependent phase parameter is nonzero. In particular, we find that the inphase state can be globally attracting. This is evident from Fig. 7, though it is impossible to deduce from a knowledge of $\sigma$ alone whether the dynamics is $\mathrm{CW}$ or some more complicated inphase state.

Finally, we present in Fig. 8 the results from a more extensive set of simulations in which we map out the situation in the $\zeta-\theta$ plane. The left panel displays the value of $\mu_{\max }$ for the matrix (13) and the right panel displays $\sigma$ determined from numerical simulations. The results are seen to be more or less independent of $\theta$ as long as $\theta$ is bigger than about 1 . There is a broad strip over which the synchronization is very high, and this tracks closely the region over which $\mu_{\max }<1$, i.e., where the inphase state is calculated to be locally stable. 


\section{ACKNOWLEDGMENT}

The authors thank H. Brusselbach, M. Minden, and W. Ray for useful discussions. This work was supported by the High Energy Laser Joint Technology Office and the US Army Research Office under Award No. W911NF-05-1-0506. Any opinions, findings, and conclusions or recommendations expressed in this publication are those of the authors and do not necessarily reflect the views of the High Energy Laser Joint Technology Office or the Army Research Office.

\section{REFERENCES}

[1] T. Simpson, A. Gavrielides, and P. Peterson, "Extraction characteristics of a dual fiber compound cavity," Opt. Exp., vol. 10, no. 20, pp. 1060-1073, 2002.

[2] A. Shirakawa, T. Saitou, T. Sekiguchi, and K. Ueda, "Coherent addition of fiber lasers by use of a fiber coupler," Opt. Exp., vol. 10, no. 21, pp. 1167-1172, 2002

[3] D. Sabourdy, V. Kermène, A. Desfarges-Berthelemot, L. Lefort, A. Barthélémy, C. Mahodaux, and D. Pureur, "Power scaling of fibre lasers with all-fibre interferometric cavity," Electron. Lett., vol. 38, no. 14, pp. 692-693, 2002.

[4] D. Sabourdy, V. Kermene, A. Desfarges-Berthelemot, L. Lefort, A. Barthelemy, P. Even, and D. Pureur, "Efficient coherent combining of widely tunable fiber lasers," Opt. Exp., vol. 11, no. 2, pp. 87-97, 2003.

[5] M. L. Minden, H. W. Bruesselbach, J. L. Rogers, M. S. Mangir, D. C. Jones, G. J. Dunning, D. L. Hammon, and A. J. Solis, "Self-organized coherence in fiber laser arrays," Proc. SPIE, vol. 5335, pp. 89-97, 2004.

[6] V. A. Kozlov, J. Hernández-Cordero, and T. F. Morse, "All-fiber coherent beam combining of fiber lasers," Opt. Lett., vol. 24, no. 24, pp. 1814-1816, 1999.

[7] N. M. Lyndin, V. A. Sychugov, A. E. Tikhomirov, and A. A. Abramov, "Laser system composed of several active elements connected by singlemode couplers," Quantum Electron., vol. 24, no. 12, pp. 1058-1061, 1994.

[8] H. Bruesselbach, D. C. Jones, M. S. Mangir, M. L. Minden, and J. L. Rogers, "Self-organized coherence in fiber laser arrays," Opt. Lett., vol. 30, no. 11, pp. 1339-1341, 2005.

[9] H. Bruesselbach, M. L. Minden, J. L. Rogers, D. C. Jones, and M. S. Mangir, "200 W self-organized coherent fiber arrays," presented at the Conf. Lasers Electro-Opt./Quantum Electron. Laser Sci. Photon. Appl. Syst. Techol., Tech. Dig. (CD), Baltimore, MD, Paper CMDD4, 2005.

[10] J. L. Rogers, S. Peles, and K. Wiesenfeld, "Model for high-gain fiber laser arrays," J. Quantum Electron., vol. 41, no. 6, pp. 767-773, 2005.

[11] S. Peles, J. L. Rogers, and K. Wiesenfeld, "Synchronization in fiber laser arrays: Theoretical study," Proc. SPIE, vol. 5971, pp. 59 710R-1-59 710R-10, 2005.

[12] S. Peles, J. L. Rogers, and K. Wiesenfeld, "Robust synchronization in fiber laser arrays," Phys. Rev. E, Stat. Phys. Plasmas Fluids Relat. Interdiscip. Top, vol. 73, no. 2, pp. 026212-1-026212-14, 2006.

[13] W. Ray, K. Wiesenfeld, and J. L. Rogers, "Refined fiber laser model," Phys. Rev. E, Stat. Phys. Plasmas Fluids Relat. Interdiscip. Top., vol. 78, no. 4, pp. 046203-1-046203-10, 2008.

[14] A. E. Siegman. (2004). Resonant modes of linearly coupled multiple fiber laser structures [Online]. Available: http://www.stanford.edu/ siegman/ coupled_fiber_modes.pdf

[15] D. Kouznetsov, J. Bisson, A. Shirakawa, and K. Ueda, "Limits of coherent addition of lasers: Simple estimate," Opt. Rev., vol. 12, no. 6, pp. 445-447, 2005.

[16] J. E. Rothenberg, "Passive coherent phasing of fiber laser arrays," Proc. SPIE, vol. 6873, pp. 687 315-1-687 315-9, 2008.
[17] H. Bruesselbach, S. Wang, M. L. Minden, D. C. Jones, and M. S. Mangir, "Power-scalable phase-compensating fiber-array transceiver for laser communications through the atmosphere," J. Opt. Soc. Amer. B, Opt. Phys., vol. 22, no. 2, pp. 347-353, 2005.

[18] W. Ray, K. Wiesenfeld, and J. L. Rogers,, "Coherence between two coupled lasers from a dynamics perspective," Opt. Exp., submitted for publication.

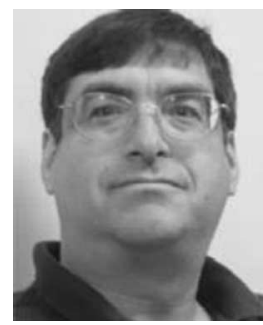

Kurt Wiesenfeld received the B.S. degree from Massachusetts Institute of Technology, Cambridge, in 1979, and the Ph.D. degree from the University of California (UC) at Berkeley, Berkeley, in 1985, both in physics.

After postdoctoral positions at UC Santa Cruz and Brookhaven National Laboratory, Upton, NY, he joined the physics faculty at Georgia Institute of Technology, Atlanta, in 1987. His current research interests lie in the application of dynamical systems theory to various problems in fundamental and applied physics, including synchronization phenomena, avalanching systems, and the beneficial effects of noise (stochastic resonance).

Dr. Wiesenfeld is a Member of the Society for Industrial and Applied Mathematics, and a Fellow of the American Physical Society.

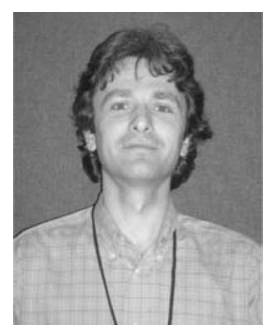

Slaven Peles received the B.Sc. degree from the University of Sarajevo, Sarajevo, Bosnia and Herzegovina, and the Ph.D. degree from the University of Manitoba, Winnipeg, MB, Canada, both in physics.

During 2001-2005, he was with Georgia Institute of Technology, Atlanta, as a Joseph Ford Fellow. From 2006 to 2007, he was with the University of California, Santa Barbara, as a Specialist. He is currently a Senior Research Engineer at United Technologies Research Center, East Hartford, CT. His current research interests include complex systems modeling, power electronics, fuel cell technology, and nonlinear dynamics.

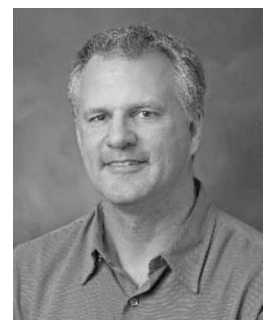

Jeffrey L. Rogers received the B.S. degree in physics and the M.S. degree from Florida Atlantic University, Boca Raton, in 1992 and 1994, respectively, and the M.S. degree from Emory University, Atlanta, GA, in 1996, and the Ph.D. degree from Georgia Institute of Technology, Atlanta, in 2001, both in physics.

He initially joined HRL Laboratories in 2001 as a Postdoctoral Fellow. He has been a Robert G. Shackleford Fellow and a Motorola Fellow. He joined California Institute of Technology, Pasadena, as a Visiting Faculty of Control and Dynamical Systems in 2006 while he was a Research Scientist at HRL Laboratories. His current research interests include a range of topics in nonlinear sciences, optics, electronics, computation (classical, dynamical, and quantum), statistical mechanics, and systems far-from-equilibrium. 